\title{
QUALIDADE DE VIDA DE MULHERES CLIMATÉRICAS DE ACORDO COM O ESTADO MENOPAUSAL
}

\author{
Ronilson Ferreira FREITAS ${ }^{1}$ \\ Tahiana Ferreira FREITAS ${ }^{2}$ \\ Débora Ribeiro VIEIRA ${ }^{3}$ \\ Natália Gonçalves Santana ROCHA ${ }^{3}$ \\ Gustavo Souza SANTOS ${ }^{4}$ \\ Vivianne Margareth Chaves Pereira REIS ${ }^{5}$ \\ Betânia Maria Araújo PASSOS \\ Josiane Santos Brant ROCHA ${ }^{7}$
}

\footnotetext{
${ }^{1}$ Mestrando em Saúde, Sociedade e Ambiente pela Universidade Federal dos Vales do Jequitinhonha e Mucuri - UFVJM. ronnypharmacia@gmail.com

${ }^{2}$ Graduanda em Fonoaudiologia pelas Faculdades de Saúde Ibituruna - FASI.

${ }^{3}$ Graduanda em Medicina pelas Faculdades Integradas Pitágoras de Montes Claros - FIPMoc.

${ }^{4}$ Graduado em Comunicação Social pelas Faculdades Integradas Pitágoras de Montes Claros - FIPMoc.

${ }^{5}$ Mestre em Atividades Físicas e Desportivas pela Universidade de Trás-os-Montes e Alto Douro -

UTAD.

${ }^{6}$ Doutora em Ciências do Desporto pela Universidade de Trás-os-Montes e Alto Douro - UTAD.
}

Recebido em: 08/11/2014 - Aprovado em: 05/06/2015 - Disponibilizado em: 15/07/2015

\section{RESUMO}

Objetivo: Comparar a qualidade de vida de mulheres climatéricas de acordo com o estado menopausal. Metodologia: Trata-se de um estudo quantitativo, do tipo descritivo e transversal de base populacional, incluindo 626 mulheres com idades compreendidas entre os 40 e os 65 anos, que responderam um formulário sócio demográfico e clínico, a qualidade de vida foi avaliada através da Escala de Avaliação da Menopausa (Menopause Rating Scale- MRS). O teste $t$ de Student foi utilizado na comparação das médias dos grupos, tendo sido considerado um nível de significância estatística de $p<0,05$. Resultados: A análise comparativa das médias relativas aos escores totais da MRS revelou variações significativas entre os grupos estudados, indicando que o estado menopausal interferiu nos índices de qualidade de vida das mulheres $(\mathrm{p}<0,05)$. Observou-se que as pacientes em menopausa induzida apresentaram o pior escore $(18,26)$ de qualidade de vida em comparação com os outros dois grupos $(\mathrm{p}<0,05)$, bem como as mulheres em menopausa natural mostraram maior comprometimento da qualidade de vida, mostrado pelo escore elevado $(15,28)$, em comparação às entrevistadas que não estavam na menopausa, as quais apresentaram escore médio de 11,38. Conclusão: Conclui-se que mulheres com menopausa induzida tendem a ter piores escores de qualidade de vida quando comparadas com mulheres na pré-menopausa e com menopausa natural.

Descritores: Qualidade de Vida, Estado Menopausal, Climatério.

\section{Quality of life of women under the weather menopausal status}

\section{ABSTRACT}

Objective: To compare the quality of life of menopausal women according to menopausal status. Methodology: This study was a quantitative, descriptive and cross-sectional population-based, including 626 women aged between 40 and 65 years who completed a demographic and clinical social form, quality 
of life was assessed by Menopause Rating Scale (Menopause Rating Scale-MRS). The Student t test was used to compare the means of the groups, and was considered a statistical significance level of $\mathrm{p}<0.05$. Results: Comparative analysis of the averages for the total scores of the MRS revealed significant variations between groups, indicating that the menopausal state interfered in the indices of quality of life of women ( $\mathrm{p}<0.05)$. It has been observed that patients induced menopause showed the lowest score (18.26) quality of life compared with the other two groups ( $p<0.05)$, as well as natural menopause in women showed greater impairment of the quality of life, shown by the high score (15.28), compared to respondents who were not menopausal, which showed a mean score of 11.38. Conclusion: We conclude that women with induced menopause tend to have worse health-related quality of life when compared with premenopausal women with natural menopause.

Keywords: Quality of Life, Menopausal Status, Climacteric.

\section{INTRODUÇÃO}

O período denominado de climatério compreende um declínio gradual da função hormonal ovariana que ocorre fisiologicamente na mulher, sendo este período seguido da instalação da menopausa e o encerramento da fase reprodutiva feminina. Comumente, as alterações hormonais oriundas

do

hipoestrogenismo, que caracteriza essa fase, desencadeiam desde sintomas vasomotores, até alterações psicológicas, urogenitais, sexuais, distúrbios do sono entre outros (NELSON, 2008).

O Brasil tem passado por alterações em sua composição populacional, e tal característica tem se tornado cada vez mais evidente, principalmente no que se refere ao aumento do número de mulheres em comparação ao de homens no país, e do crescente envelhecimento populacional, sendo que a faixa etária que se situa acima dos 60 anos nunca foi tão expressiva. Tal cenário acarretou em um interesse crescente acerca das questões relacionadas ao climatério e suas repercussões na saúde e na qualidade de vida feminina (SOBRAC, 2003; DE LORENZI; BARACAT, 2005).

A qualidade de vida é definida pela Organização Mundial de Saúde como uma concepção pessoal do indivíduo, que se baseiam no seu contexto sociocultural, valores, sonhos, metas, padrões e apreensões para caracterizar sua posição na vida (OMS, 1994). Assim, a qualidade de vida das mulheres no climatério sofre influência tanto pela presença, quanto pela severidade dos sintomas decorrentes do declínio estrogênico como por outros fatores de ordem emocional e cultural que estão ligados ao próprio envelhecimento (DE LORENZI et al., 2006).

A percepção desse fator, no entanto, ainda é muito carente de investigações cientificas no país, pouco se conhece acerca das implicações da 
menopausa na qualidade de vida feminina, havendo a indicação na literatura que novos estudos sejam realizados nessa área a fim de suprir tal lacuna no conhecimento (HEINEMANN; $\quad$ POTTHOFF; SCHNEIDER, 2003). Neste contexto, este estudo visou comparara qualidade de vida de mulheres climatéricas de acordo como estado menopausal.

\section{METODOLOGIA}

Trata-se de um estudo quantitativo, do tipo transversal, ocorrido no período de junho a outubro de 2013. A amostra foi constituída por 626 mulheres entre 40 a 65 anos de idade, no período climatérico, selecionadas aleatoriamente. Selecionou-se por conglomerado, 10 unidades de Estratégias de Saúde da Família, dentre as 63 que assistem a cidade de Montes Claros, Minas Gerais. Foram excluídas da pesquisa mulheres que apresentaram transtornos mentais ou algum comprometimento que as dificultassem responder às avaliações propostas.

Inicialmente, aplicou-se um formuláriopara avaliar as características sócio demográficas e clínicas, na qual se destacam: faixa etária, nível de atividade física e tipo de menopausa.Os dados antropométricos avaliados foram: peso e altura para cálculo do IMC e circunferência abdominal. A qualidade de vida no climatério foi investigada através da Escala de Avaliação da Menopausa (Menopause Rating Scale MRS), escala esta utilizada em uma população específica, mulheres no climatério,validado e reconhecido parauso no Brasil (HEINEMANN et al., 2004), constituída por onze itens que avaliam os sintomas climatéricos, distribuídos por três subescalas: (a) Somática - falta de ar, suores, calores; mal-estar do coração; problemas de sono; e problemas musculares e nas articulações (itens 1-3 e 11, respectivamente); (b) Psicológica Estado de ânimo depressivo; irritabilidade; ansiedade; e esgotamento físico e mental (itens 4-7, respectivamente); e (c) Urogenital Problemas sexuais; problemas de bexiga; e ressecamento vaginal (itens 810, respectivamente). Cada item pode ser graduado de 0 a $4(0=$ nenhum; $1=$ pouco severo; $2=$ moderado $; 3=$ severo; $4=$ muito severo). O score da escala total resulta da soma dos scores das três subescalas e os sintomas podem classificar-se em a) assintomáticos ou escassos (0-4 pontos), b) leves (5-8 pontos), c) moderados (9-15 pontos) ou d) severos (mais de 16 pontos). De 
acordo com a pontuação da subescala de sintomas somáticos, estes podem ser classificados em a) assintomáticos ou escassos (dois pontos ou menos), b) leves (3-4 pontos), c) moderados (5-8 pontos), ou d) severos (mais de 9 pontos). Os sintomas psicológicos serão igualmente classificados, de acordo com a pontuação, em a) assintomáticos ou escassos (um ponto ou menos), b) leves (2-3 pontos), c) moderados (4-6 pontos) ou d) severos (mais de 7 pontos). Por último, os sintomas urogenitais classificam-se em a) assintomáticos ou escassos ( 0 pontos $),$ b) leves (1 ponto), c) moderados (2-3 pontos) ou d) severos (4 pontos ou mais).

Os dados foram analisados com o programa SPSS (versão 20.0; Armonk, New York, USA), tendo sido considerado um nível de significância estatística de $\mathrm{p}<0,05$. O teste $t$ de Student foi utilizado na comparação dos grupos.

A pesquisa seguiu todos os critérios estabelecidos pela Resolução número 466/2012 do Conselho Nacional de Saúde para pesquisas envolvendo seres humanos. As participantes foram previamente esclarecidas sobre os objetivos e procedimentos referentes à pesquisa e assinaram o Termo de Consentimento Livre e Esclarecido anterior à coleta de dados, tendo sido aprovado pelo Comitê de Ética e Pesquisa da Universidade Estadual de Montes Claros - Unimontes, sob o parecer de número 311.628/2013.

\section{RESULTADOS}

O presente estudo avaliou 626 pacientes, sendo que a 59,3\% destas declararam ter idade entre 45 e 59 anos, e $61,1 \%$ das mulheres relataram ter menopausa do tipo natural. Quanto aos níveis de atividade física, somente $22,2 \%$ das entrevistadas confirmaram praticar atividade física regular. Já em relação IMC e CA, observou-se que $38 \%$ estavam com sobrepeso, enquanto $30,4 \%$ eram obesas. Detectou-se que, $66,6 \%$ das mulheres entrevistadas mostraram risco muito elevado para ocorrência de complicações metabólicas associadas à obesidade, demonstrada pela elevada circunferência abdominal (Tabela 1). 
Tabela 1 - Perfil sócio demográfico e clinico das mulheres climatéricas

\begin{tabular}{lcc}
\hline \multicolumn{1}{c}{ Fatores } & Variáveis & $(\%)(\mathrm{n})$ \\
\hline Faixa Etária & 40 a 44 anos & $17,7(111)$ \\
& 45 a 59 anos & $59,3(371)$ \\
& 60 a 65 anos & $23,0(144)$ \\
Atividade física & Prática atividade física & \\
& Não prática atividade física & $22,2-139$ \\
& & $77,8-487$ \\
Índice de Massa Corporal & Normal (IMC 18,5 $-24,99)$ & $31,6-198$ \\
(IMC) & Sobrepeso (IMC 25,00 - 29,99) & $38,0-238$ \\
& Obesidade (IMC $\geq 30,00)$ & $30,4-190$ \\
Circunferência abdominal (CA) & Rem risco (menos que $80 \mathrm{~cm})$ & $11,0-69$ \\
& Risco aumentado $(>80 \mathrm{~cm})$ & $22,4-140$ \\
Tipo de menopausa & Pré-menopausa & $66,6-418$ \\
& Natural & $28,0-175$ \\
& Induzida (histerectomia) & $61,1-383$
\end{tabular}

Nota: (\%) - Porcentagem observada; (n) - Tamanho da Amostra.

Com relação à qualidade de vida, observa-se que as mulheres que relataram menopausa induzida e natural apresentaram escores médios maiores, 7,13 e 6,48 , respectivamente, para os sintomas somáticos, enquanto mulheres na pré-menopausa relataram apenas escores de 4,49, ( $p<0,05)$, ou seja, apresentaram sintomas em grau leve para este item na qualidade de vida. $\mathrm{Na}$ associação entre os sintomas psicológicos e o estado menopausal, obteve-se um elevado escore frente aos sintomas psicológicos $(\mathrm{p}<0,05)$ nas mulheres menopausadas de forma induzida $(7,76)$, diferindo dos escores moderados das menopausadas por forma natural e pré-menopausadas $(p=0,004)$ que são 6,40 e $\quad 5,65$, respectivamente. $\mathrm{Na}$ analise da presença de sintomas urogenitais identificaram-se escores moderados nas pacientes menopausadas por forma natural e induzida (2,40 e 3,37 respectivamente) enquanto as pré-menopausadas adquiriram escore leve de 1,25 (Tabela 2). 
Tabela 2 - Comparação da qualidade de vida de mulheres climatéricas de acordo com o estado menopausal.

\begin{tabular}{|c|c|c|c|c|c|}
\hline \multirow[b]{2}{*}{ Sintomas } & \multicolumn{3}{|c|}{ Estado Menopausal } & \multirow[b]{2}{*}{$\mathrm{F}$} & \multirow[b]{2}{*}{$\mathrm{P}$} \\
\hline & Pré-Menopausa & $\begin{array}{c}\text { Menopausa } \\
\text { Natural }\end{array}$ & $\begin{array}{l}\text { Menopausa } \\
\text { Induzida }\end{array}$ & & \\
\hline Sintomas Vegetativos & 4,49 & 6,48 & 7,13 & 19,16 & ,000 \\
\hline Sintomas Psicológicos & 5,65 & 6,40 & 7,76 & 5,70 & ,004 \\
\hline Sintomas Urogenitais & 1,25 & 2,40 & 3,37 & 21,00 &, 000 \\
\hline MRS & 11,38 & 15,28 & 18,26 & 19,28 &, 000 \\
\hline
\end{tabular}

A análise comparativa das médias relativas aos escores totais da MRS revelou variações significativas entre os grupos estudados, indicando que o estado menopausal interferiu nos índices de qualidade de vida das mulheres $(\mathrm{p}<0,05)$. Observou-se que as pacientes em menopausa induzida apresentaram o pior escore $(18,26)$ de qualidade de vida em comparação com os outros dois grupos $(\mathrm{p}<0,05)$, bem como as mulheres em menopausa natural mostraram maior comprometimento da qualidade de vida, mostrado pelo escore elevado $(15,28)$, em comparação às entrevistadas que estavam na pré-menopausa, as quais apresentaram escore médio de 11,38.

\section{DISCUSSÃO}

No presente estudo, foram avaliadas 626 mulheres atendidas em Unidades Básicas de Saúde da cidade de Montes Claros, Minas Gerais, objetivando comparar a qualidade de vida e o estado menopausal de mulheres climatéricas.

Diversos fatores determinam a idade em que a menopausa de forma natural ocorre, entre eles podemos destacar o estilo de vida e hábitos alimentares, fatores estes passíveis de modificações para uma menopausa tardia e minimização dos sintomas climatéricos, os quais comprometem a qualidade de vidas das mulheres que o experimentam. Dentre os hábitos de vida que determina a menopausa precoce pode-se enfatizar o tabagismo e os exercícios físicos vigorosos, sendo que diversos estudos realizados na atualidade apontam para o papel da alta ingestão de calorias totais, frutas e proteínas em atrasar a menopausa natural, enquanto que a elevada ingestão de gordura polisaturada é capaz de acelerar este estado menopausal (MALHEIROS et al., 2014; SAPRE; THAKUR, 2014). 
$\mathrm{Na}$ fase climatérica há alterações endócrinas decorrentes do declínio da atividade ovariana, com mudanças biológicas em função da diminuição da fertilidade e mudanças clínicas consequentes das alterações do ciclo menstrual (Gallon, 2009). Assim, esse novo perfil fisiológico torna o individuo mais susceptível ao aumento de peso e da gordura abdominal, gerando uma associação positiva entre estes fatores e a doença cardiovascular (SILVA, 2010), salientando que os dados obtidos na análise comportamental do presente estudo, que ressaltaram a prevalência de obesidade, sobrepeso e alto riso de ocorrência de complicações metabólicas associadas a um perfil lipídico corporal desvantajoso épreocupante. Esse resultado negativo do perfil comportamental das pesquisadas vai de acordo com os resultados de pesquisas como a de Colpani et al., (2014) confirmando a situação alarmante desse grupo populacional e da necessidade de um acompanhamento e medidas intervencionistas mais eficazes em âmbito de atenção básica a saúde para mudança desse quadro.

$\mathrm{O}$ grupo estudado nesta pesquisa foi composto por um maior número de mulheres na pós-menopausa, sendo que destas a maioria relatou a ocorrência da menopausa por forma natural.
Observou-se associação entre os sintomas que comprometem a qualidade de vida no climatério e a variável estadomenopausal, sendo quedurante a menopausa, os sintomas vasomotores são uma das principais queixas que levam as mulheres a procurarem ajuda médica (CARRANZA-LIRA, 2009).

A queda de estrogênio na menopausa tem íntima relação com a ocorrência dos sintomas vasomotores, como suores, calor ("fogachos"), além de sugerir que este hormônio exerça efeitos cardioprotetores. Estudos demonstraram que mulheres na prémenopausa têm menor risco para doença cardiovascular em comparação com os homens, mas a incidência de doenças cardiovasculares em mulheres aumenta após a menopausa, o que também contribui para a severidade dos sintomas vasomotores em mulheres nesta fase de sua vida. Este dado é comprovado por trabalhos que mostram que a reposição hormonal reduziu o risco para a ocorrência das doenças cardiovasculares ao atrasar ou melhorar o desenvolvimento da placa aterosclerótica (MURPHY; STEENBERGEN, 2014) e redução dos sintomas somáticos (MAMERI FILHO et al., 2005), o que altera a qualidade de vida destas mulheres. 
Verificou-se forte relação entre os sintomas psicológicos e o estado menopausal nas pesquisadas, fato que vai de acordo com os achados de Rauma et al., (2013), onde 55\% das mulheres pesquisadas relataram o uso de antidepressivos e $29 \%$ uso de outros medicamentos psicoativos. A alta utilização e história da pensão por invalidez devido à causa psiquiátrica foram associadas com uma probabilidade muito maior de relatar o uso de medicação psicoativa nesta fase da vida da mulher. Ainda, estudo realizado por Chen et al., (2013) suporta uma associação entre a transição da menopausa sintomática e transtorno depressivo em mulheres de meia-idade, e um risco aumentado de depressão com comorbidades médicas.

Além dos sintomas vasomotores (ondas de calor), os atróficos (principalmente a atrofia vaginal) foram relatados pelas mulheres pesquisadas. Esses sintomas urogenitais foram relacionados de modo moderado com o estado menopausal e se apresentam como consenso da decorrência da diminuição dos níveis hormonais, o que pode levar a uma perda da rugosidade da mucosa vulvo vaginal com redução acentuada da lubrificação gerando a secura genital. Tais alterações urogenitais alteram a atividade sexual das pacientes acometidas, levando a distúrbios psíquicos atribuídos a transição menopáusica. Dessa forma, há uma visão socialmente construída, que vem com uma conotação negativa de perdas, onde a menopausa é um assunto constrangedor, íntimo e relacionado à perda da sexualidade (VIGETA; BRETAS, 2004).

Estudo realizado por Cabral et $a l .$, (2012), pesquisando a influência dos sintomas climatéricos sobre a função sexual de mulheres de meia idade, demonstraram que os sintomas somatovegetativos, urogenitais e psicológicos do MRS apresentaram-se mais elevados nas mulheres com risco de disfunção sexual, sendo significativos para todas as comparações.

Entretanto, estudo realizado por De Lorenzi et al., (2005), pesquisando a qualidade de vida segundo estado menopausa em mulheres no sul do Brasil, mostrou que a qualidade de vida não sofreu influência do estado menopausal e que a maior severidade dos sintomas somato-vegetativos no grupo pós-menopáusico provavelmente se deve ao maior hipoestrogenismo nessa fase, favorecendo sintomas vasomotores e atrofia urogenital, e a maior severidade dos sintomas psicológicos entre as mulheres pré- 
menopáusicas talvez estaria relacionada

à maior preocupação acerca da menopausa e suas implicações para a saúde.

Em sua revisão literária, Gordia et al., (2011) relatam que com base na produção científica atual há indícios de que a qualidade de vida parece estar associada tanto a fatores biológicos e comportamentais quanto a aspectos sociodemográficos e culturais, porém a magnitude da interferência destes fatores sobre a qualidade de vida ainda é desconhecida.

A qualidade de vida no geral foi influenciada pelo estado menopausal das pacientes no referido estudo, com as mulheres em menopausa apresentando pior escore em relação às que relataram não atingir essa fase, demonstrado pela sintomatologia mais severa no grupo de mulheres em menopausa induzida, bem como os sintomas somatovegetativos, psicológicos e urogenitais influenciados pelo estado menopausal, mostrando piores escores em todos os domínios da qualidade de vida em comparação àquelas mulheres pré-menopausadas. Porém, salienta-se a necessidade de estudos de intervenção que possibilite matitudes mais claras que resultem namelhoria da qualidade de vida das pessoas, visto tais tipos de estudo serem escassos e relevantes (PEREIRA; TEIXEIRA; SANTOS，2012)

\section{CONCLUSÃO}

Neste estudo concluiu-se que há forte relação entre o estado menopausal e a qualidade de vida das mulheres, indo de acordo com os estudos utilizados como base cientifica de comparação. Destacou-se que a qualidade de vida representa uma variável de grande relevância social, que o meio científico já conceituou e aceitou como importantedado a ser averiguado.

$\mathrm{O}$ amparo à saúde das mulheres menopausadas configura uma ação com particularidades que devem ser atentadas pelos profissionais da área da saúde e postas em prática, visando um auxiliou eficaz a esse grupo populacional crescente. Compreender a mulher em suas mais diversas peculiaridades, no seu contexto sociocultural e no seu período menopausal configuram atitudes fundamentais para uma correta assistência a saúde da mesma impactando em seu bem-estar e expectativa de vida. 


\section{REFERÊNCIAS}

CABRAL, P. U. L. et al. Influência dos sintomas climatéricos sobre a função sexual de mulheres de meia-idade. Revista Brasileira de Ginecologia e Obstetrícia. v. 34, n.7, p.329-34, 2012.

CARRANZA-LIRA S. Safety, efficacy and patient acceptability of drospirenone and estradiol in the treatment of menopausal vasomotor symptoms: a review. ClinInterv Aging. v. 4, p. 59-62, 2009.

COLPANI, V.; SPRITZER, P. M.; LODI, A. P.; DORIGO, G. G.; MIRANDA, I. A. S.; HAHN, A. B.; PALLUDO, L. P.; PIETROSKI, R. L.; OPPERMANN, K. Atividade física de mulheres no climatério: comparação entre auto-relato e pedômetro. Revista de Saúde Pública. v. 48 n. 2, 2014.

CHEN, M. H.; SU, T. P.; LI, C. T.; CHANG, W. H.; CHEN, T. J.; BAI, Y. M. Symptomatic menopausal transition increases the risk of new-onset depressive disorder in later life: a nationwide prospective cohort study in Taiwan. PlosOne. v, 13, n. 8, 2013.

DE LORENZI, D. R. S.; BARACAT, E. C. Climatério e qualidade de vida. Femina. v. 33, p. 899-903, 2005.

DE LORENZI, D. R. S.; BARACAT, E. C.; SACILOTO, B.; PADILHA JR, I. Fatores associados à qualidade de vida após a menopausa. Revista da Associação Medica Brasileira. v. 52, n. 5, p. 312-7, 2006.

GALLON, C. W. Perfil nutricional e qualidade de vida de mulheres no climatério [dissertação]. Porto Alegre:Universidade Federal do Rio Grande do Sul; 2009.
GORDIA, A. P. et al. Qualidade de vida e saúde: conceito histórico, definição, avaliação e fatores associados. Revista Brasileira de Qualidade de Vida. v. 3, n. 1, p. 40-52, 2011.

HEINEMANN, L. A.; POTTHOFF, P.; SCHNEIDER, H. P. International versions of the Menopause Rating Scale (MRS). Health and Quality Life Outcomes. v. 1, n. 28, 2003.

HEINEMANN, K.; RUEBIG, A.; POTTHOFF, P.; SCHNEIDER, H. P. G.; STRELOW, F.; HEINEMANN, L. A. J.; THAI, D. M. The menopause rating scale (MRS): a methodological review. Health and Quality of Life Outcomes. v. 2, p. 2-45, 2004.

MALHEIROS, E. S. A.; CHEIN, M. B. C.; SILVA, D. S. M.; DIAS, C. L. L.; BRITO, L. G. O.; PINTO-NETO, A. M.; BRITO, L. M. O. Síndrome climatérica em uma cidade do Nordeste brasileiro: um inquérito domiciliar. Revista Brasileira de Ginecologia e Obstetrícia. v. 36, n. 4, p. 163-9, 2014.

MAMERI FILHO, J. et al . Efeitos da associação estro-androgênica em mulheres na pós-menopausa. Rev. Bras. Ginecol. Obstet. v. 27, n. 3, 2005.

MURPHY, E.; STEENBERGEN, C. Estrogen regulation of protein expression and signaling pathways in the heart. Biologyof Sex Differences. v. 5, n.1, 2014.

NELSON, H. D. Menopause. Lancet. v. 371, n. 9614, p. 760-70, 2008.

ORGANIZAÇÃO MUNDIAL DE SAÚDE - OMS. Avaliação da qualidade de vida: perspectivas internacionais Springer, Berlim, 1994. 
PEREIRA, E. F.; TEIXEIRA, C. S.; SANTOS, A. Qualidade de vida: abordagens, conceitos e avaliação. Revista Brasileira de Educação Física e Esporte. v. 26, n. 2, p. 241-50, 2012.

RAUMA, P. H.; KOIVUMAAHONKANEN, H.; KROER, H.; TUPPURAINEN, M. T.; KAUHANEN, J.; HONKANEN, R. J. The relationship between self-reported and registrybased data on use of psychoactive medications in postmenopausal women. Biomed Central Psychiatry. v. 13, 2013.

SAPRE, S.; THAKUR, R. Life style and dietary factors determine age at natural menopause. Journal Mid life Health. v. 5, n. 1, 2014.

SILVA, L. M. Avaliação Nutricional e Consumo Alimentar de Mulheres na Menopausa [dissertação]. Criciúma:
Universidade do Extremo Sul Catarinense; 2010.

SOCIEDADE BRASILEIRA DE CLIMATÉRIO - SOBRAC. Consenso brasileiro multidisciplinar de assistência à mulher climatérica. São Paulo; 2003.

VIGETA, S. M. G. A.; BRETAS, A. C. P. A experiência da perimenopausa e pós-menopausa com mulheres que fazem uso ou não da terapia de reposição hormonal. Caderno de Saúde Pública. v. 20, n. 6, 2004. 